McAuley et al. Online Supplementary Material

\title{
Six-months of hybrid closed-loop versus manual insulin delivery with finger-prick blood glucose monitoring in adults with type 1 diabetes: a randomized, controlled trial
}

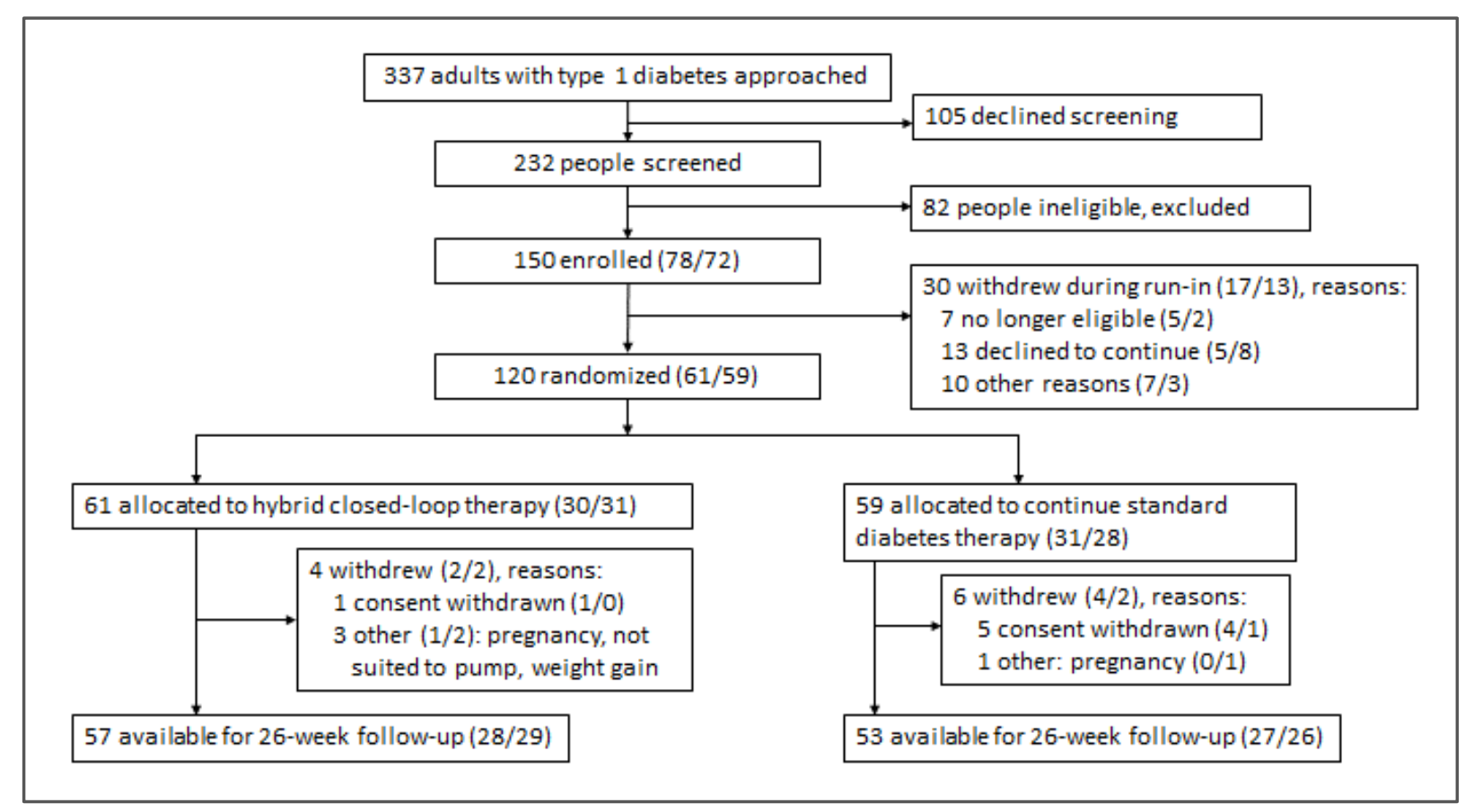

Figure S1: Trial profile. Overall numbers (baseline insulin delivery via multiple daily injections / pump). 


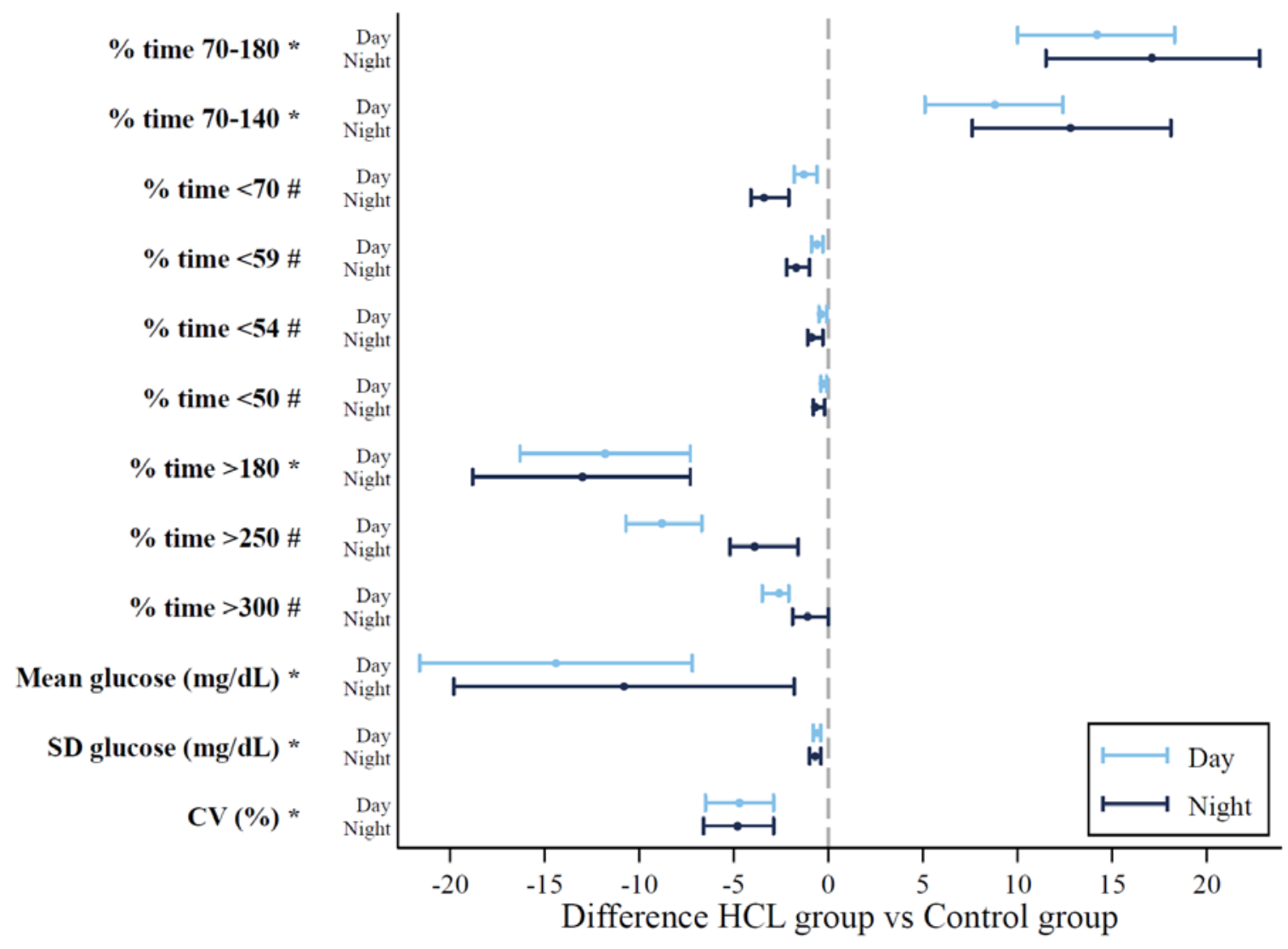

Figure S2: Sub-group analysis by Day (6am-midnight) vs Night (midnight-6am). Forest plot of differences in sensor glucose metrics at study-end between participants assigned to hybrid closed-loop (HCL) intervention versus control, presented by time of day (Day, light blue; Night, navy). Glucose levels are expressed in $\mathrm{mg} / \mathrm{dL}$ * $*$ Lines represent mean difference with adjustment for baseline values (95\% CI). \# Lines represent median difference (95\% CI). 

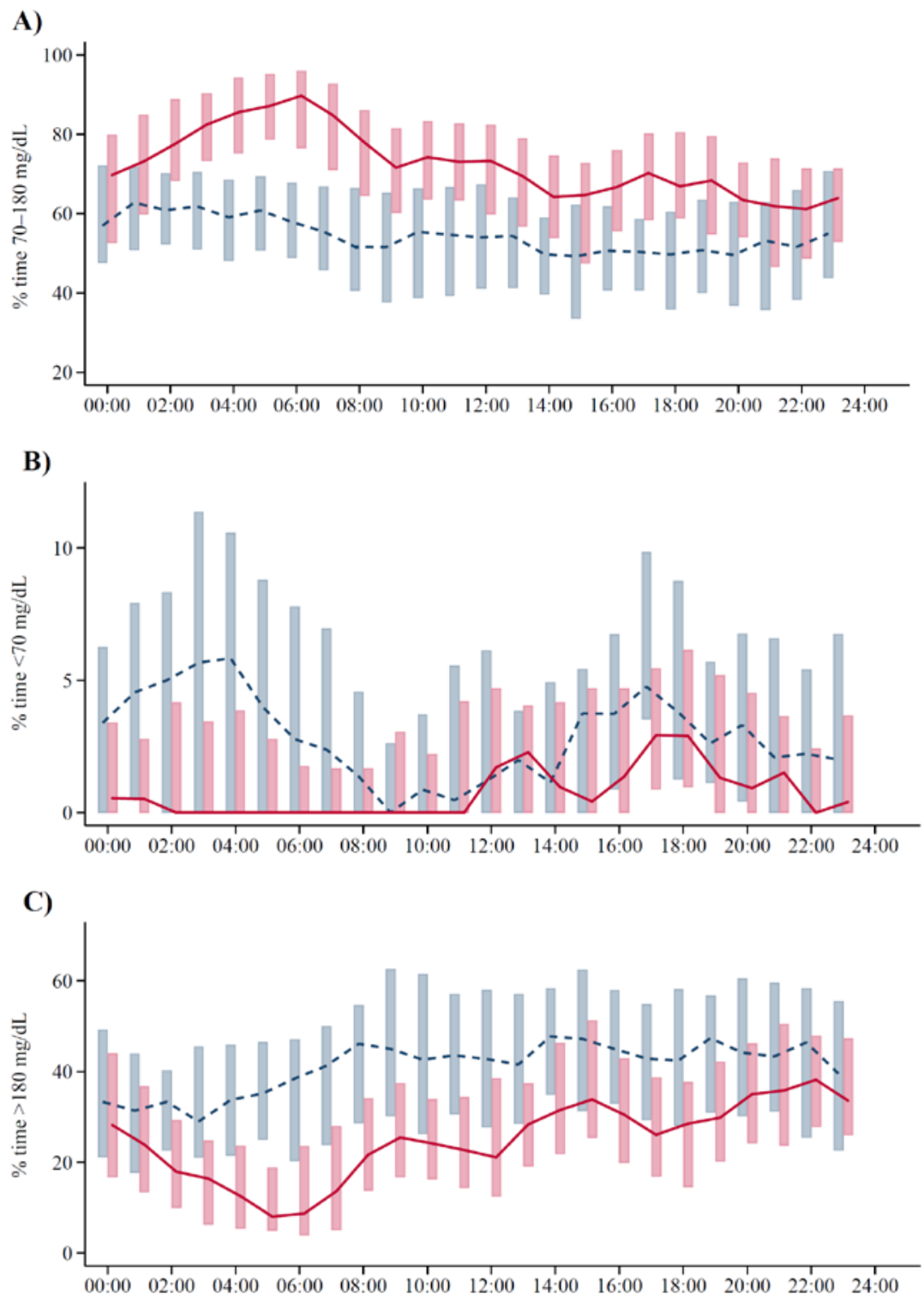

D)

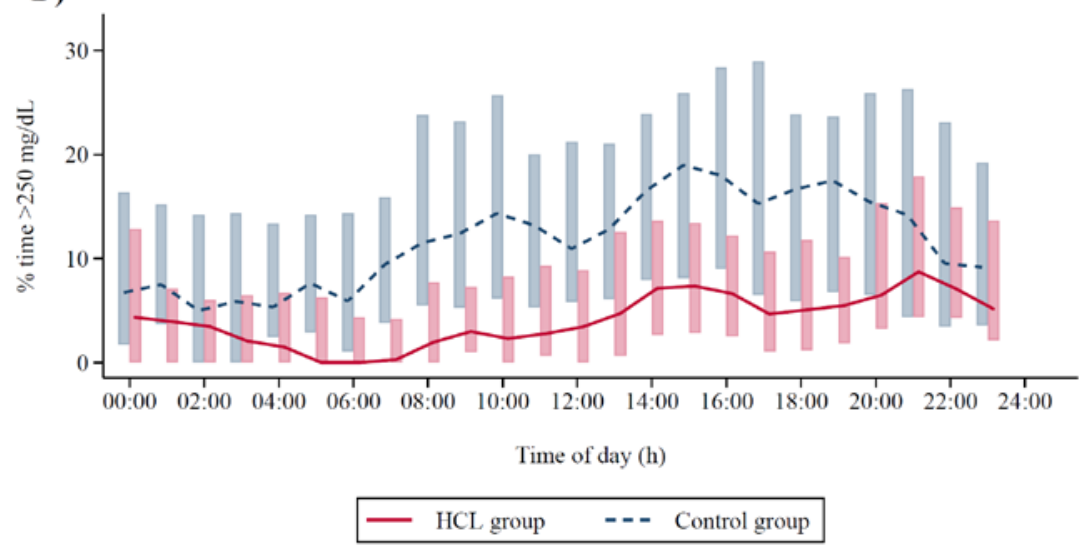

Figure S3: Proportion of continuous glucose monitoring time: in range $\mathbf{7 0 - 1 8 0 ~} \mathbf{~ m g} / \mathbf{d L}$ (Panel A), below $70 \mathrm{mg} / \mathrm{dL}$ (Panel B), above $180 \mathrm{md} / \mathrm{dL}$ (Panel C), and above $250 \mathrm{mg} / \mathrm{dL}$ (Panel D). The time is shown in hourly blocks, by allocated group (hybrid closed-loop [HCL] group, red; control group, blue). Lines represent the medians, and the bottom and top of each box represent the $25^{\text {th }}$ and $75^{\text {th }}$ percentiles. 

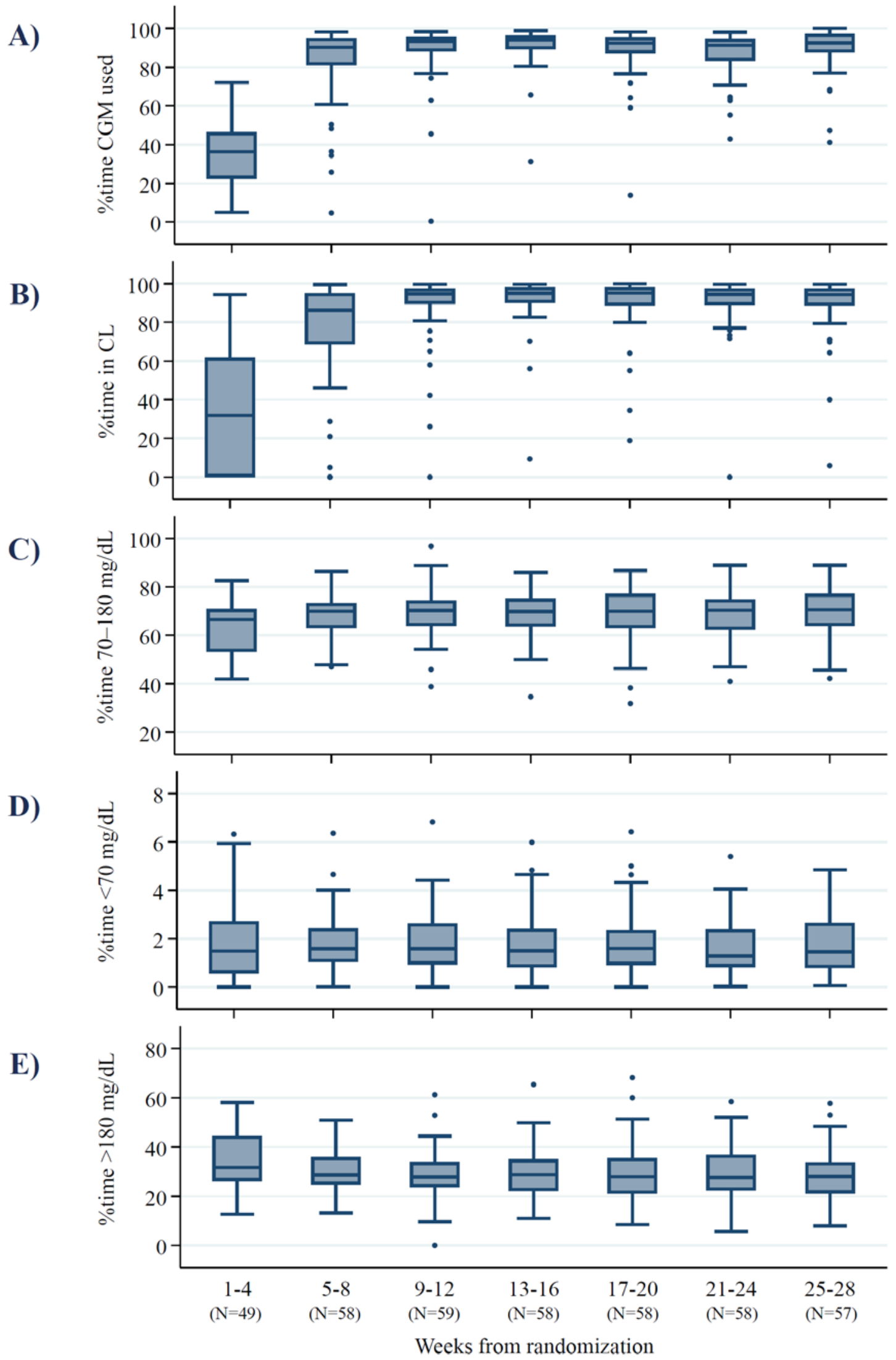

Figure S4: Intervention group - device wear and continuous glucose monitoring (CGM) metrics. Boxplots represent 4-week time blocks, starting from randomization. Glucose levels are expressed in $\mathrm{mg} / \mathrm{dL}$. CL, closed-loop. 


\section{Inclusion criteria}

An individual is eligible for inclusion in the study if ALL the following criteria are met:

1. Type 1 diabetes (diagnosis consistent with American Diabetes Association Classification of Diabetes Mellitus) for at least 1 year, with fasting C-peptide $<0.1 \mathrm{nmol} / \mathrm{L}$ (in the absence of hypoglycaemia)

2. Insulin regimen either:

o Multiple daily injections (MDI) with $\geq 4$ injections per day (including $\geq 3$ rapid-acting insulin injections and $\geq 1$ long-acting insulin injection); or

o Insulin pump therapy (CSII) established for $\geq 3$ months

3. Age 25-70 years inclusive at time of screening

4. $\mathrm{HbA}_{1 \mathrm{c}} \leq 10.5 \%$

5. Living in an area with internet and cellular phone coverage

6. $\quad$ English speaking

\section{Exclusion criteria}

An individual will be excluded from the study if ANY of the following criteria are met:

1. Chronic kidney disease (eGFR $\left.<45 \mathrm{~mL} / \mathrm{min} / 1.73 \mathrm{~m}^{2}\right)$

2. Current use of real-time CGM (defined as real-time CGM use $>25 \%$ of the time for the past 3 months)

3. Use of any non-insulin glucose-lowering agent within the past 3 months

4. Oral or injected steroid use within the past 3 months

5. Pregnancy, or planned pregnancy within study period

6. Uncontrolled coeliac disease (not following a gluten free diet), or other untreated malabsorption

7. Uncontrolled thyroid disease

8. Clinically-significant gastroparesis

9. Uncontrolled hypertension (diastolic BP >100 mmHg and/or systolic BP >160 mmHg)

10. History of myocardial infarction, severe uncontrolled heart failure, unstable angina, transient ischaemic attack, stroke, or thromboembolic disease in the past 3 months

11. Poor visual acuity precluding use of the investigational technology

12. Inability or unwillingness to meet protocol requirements (such as carbohydrate-counting, frequency of blood glucose monitoring, CGM wear as per allocated study group only)

13. Severe or unstable medical or psychological condition which, in the opinion of the investigator, would compromise the ability to meet protocol requirements 
Table S2: Missing data for study outcomes

\begin{tabular}{|c|c|c|c|}
\hline & Overall missing & $\begin{array}{c}\text { HCL group (MDI/Pump) } \\
\qquad n=61(30 / 31)\end{array}$ & $\begin{array}{c}\text { Control group (MDI/Pump) } \\
\qquad n=59(31 / 28)\end{array}$ \\
\hline CGM mid-study & 11 & $4(1 / 3)$ & $7(5 / 2)$ \\
\hline CGM study-end & 20 & $6(3 / 3)$ & $14(9 / 5)$ \\
\hline $\mathrm{HbA}_{1 \mathrm{c}}$ mid-study & 8 & $3(0 / 3)$ & $5(4 / 1)$ \\
\hline $\mathrm{HbA}_{1 \mathrm{c}}$ study-end & 9 & $2(1 / 1)$ & $7(5 / 2)$ \\
\hline ICR mid-study & 11 & $3(0 / 3)$ & $8(5 / 3)$ \\
\hline ICR study-end & 16 & $3(2 / 1)$ & $13(8 / 5)$ \\
\hline Weight mid-study & 14 & $4(0 / 4)$ & $10(5 / 5)$ \\
\hline Weight study-end & 12 & $3(2 / 1)$ & $9(4 / 5)$ \\
\hline Total daily insulin mid-study & 11 & $3(0 / 3)$ & $8(5 / 3)$ \\
\hline Proportions daily insulin mid-study & 11 & $3(0 / 3)$ & $8(5 / 3)$ \\
\hline Total daily insulin study-end & 14 & $3(2 / 1)$ & $11(6 / 5)$ \\
\hline Proportions daily insulin study-end & 14 & $3(2 / 1)$ & $11(6 / 5)$ \\
\hline 1,5-anhydroglucitol study-end & 10 & $2(1 / 1)$ & $8(5 / 3)$ \\
\hline Psychosocial study-end & 13 & $3(1 / 2)$ & $10(6 / 4)$ \\
\hline
\end{tabular}

MDI, multiple daily injections. CGM, continuous glucose monitoring. HCL, hybrid closed loop. ICR, insulin-to-carbohydrate ratio. 
Table S3: Glucose outcomes 24 hours/day at mid-study

\begin{tabular}{|c|c|c|c|c|}
\hline & $\begin{array}{l}\text { HCL group } \\
\quad(n=61)\end{array}$ & $\begin{array}{c}\text { Control group } \\
\qquad(n=59)\end{array}$ & $\begin{array}{c}\text { Difference } \mathrm{HCL} \\
\text { minus control }\end{array}$ & $p$ value \\
\hline \%time glucose $70-180 *$ & $67.8(10.9)$ & $54.9(14.4)$ & $12.4(8.5,16.3)$ & $<0.001$ \\
\hline \%time glucose $70-140 *$ & $44.3(8.2)$ & $33.7(12.1)$ & $9.1(5.8,12.4)$ & $<0.001$ \\
\hline$\%$ time glucose $<70 \dagger$ & $1.7(1.0,2.9)$ & $4.0(2.8,6.0)$ & $-2.2(-2.8,-1.3)$ & $<0.001$ \\
\hline$\%$ time glucose $<59 \dagger$ & $0.5(0.3,1.2)$ & $1.9(0.6,2.8)$ & $-1.2(-1.6,-0.6)$ & $<0.001$ \\
\hline$\%$ time glucose $<54 \dagger$ & $0.3(0.1,0.6)$ & $1.0(0.3,1.9)$ & $-0.7(-1.0,-0.3)$ & $<0.001$ \\
\hline$\%$ time glucose $<50 \dagger$ & $0.2(0.0,0.5)$ & $0.8(0.2,1.4)$ & $-0.5(-0.7,-0.2)$ & $<0.001$ \\
\hline \%time glucose $>180 *$ & $30.0(11.2)$ & $39.9(16.4)$ & $-9.0(-13.1,-4.9)$ & $<0.001$ \\
\hline$\%$ time glucose $>200 \dagger$ & $6.1(4.5,9.6)$ & $15.3(7.1,18.9)$ & $-7.1(-9.8,-3.8)$ & $<0.001$ \\
\hline$\%$ time glucose $>250 \dagger$ & $1.9(0.6,3.8)$ & $4.9(0.9,7.1)$ & $-2.0(-3.5,-0.6)$ & 0.001 \\
\hline Mean glucose * & $157(14)$ & $171(23)$ & $-11(-16,-4)$ & 0.003 \\
\hline $\mathrm{SD} *$ & $54(11)$ & $67(13)$ & $-9.0(-13,-5)$ & $<0.001$ \\
\hline $\mathrm{CV} *$ & $34.7(4.5)$ & $39.3(5.3)$ & $-3.2(-5.0,-1.4)$ & 0.001 \\
\hline $\mathrm{HbA}_{1 \mathrm{c}}(\%) *$ & $7.0(0.5)$ & $7.5(0.8)$ & $-0.4(-0.6,-0.2)$ & $<0.001$ \\
\hline $\mathrm{HbA}_{1 \mathrm{c}}(\mathrm{mmol} / \mathrm{mol}) *$ & $53(6)$ & $58(9)$ & $-4(-7,-2)$ & $<0.001$ \\
\hline
\end{tabular}

Glucose levels are expressed in mg/dL. HCL, hybrid closed loop.

* Results presented as mean (SD), mean difference (95\% CI), analysis using ANCOVA with adjustment for baseline value

† Results presented as median (IQR), median difference (95\% CI), analysis using rank sum test. 
Table S4: Intervention group continuous glucose monitoring metrics, before and after the initial HCL activation

\begin{tabular}{|c|c|c|c|c|c|c|c|}
\hline & \multirow{2}{*}{$\begin{array}{l}\text { Before closed- } \\
\text { loop activation * }\end{array}$} & \multicolumn{6}{|c|}{ After closed-loop activation } \\
\hline & & Month 1 & Month 2 & Month 3 & Month 4 & Month 5 & Month $6 \dagger$ \\
\hline Number of participants $\ddagger$ & 60 & 60 & 59 & 58 & 58 & 56 & 49 \\
\hline \% time glucose $70-180$ & $62(49,69)$ & $71(64,74)$ & $69(65,74)$ & $70(63,76)$ & $69(63,77)$ & $71(63,76)$ & $73(65,78)$ \\
\hline \% time glucose $70-140$ & $34(26,43)$ & $42(39,49)$ & $43(39,47)$ & $43(39,50)$ & $43(37,52)$ & $45(37,49)$ & $46(40,52)$ \\
\hline$\%$ time glucose $<70$ & $1.5(0.7,2.9)$ & $1.3(0.9,2.4)$ & $1.6(1.1,2.5)$ & $1.4(0.8,2.4)$ & $1.5(0.9,2.5)$ & $1.4(0.8,2.6)$ & $1.4(0.8,3.0)$ \\
\hline$\%$ time glucose $<59$ & $0.3(0.0,0.8)$ & $0.4(0.2,0.7)$ & $0.5(0.2,1.0)$ & $0.4(0.2,0.8)$ & $0.4(0.2,0.8)$ & $0.4(0.2,0.8)$ & $0.4(0.2,1.0)$ \\
\hline$\%$ time glucose $<54$ & $0.1(0.0,0.5)$ & $0.2(0.1,0.4)$ & $0.3(0.1,0.6)$ & $0.2(0.1,0.5)$ & $0.2(0.1,0.5)$ & $0.2(0.1,0.4)$ & $0.2(0.1,0.5)$ \\
\hline$\%$ time glucose $<50$ & $0.1(0.0,0.2)$ & $0.1(0.0,0.2)$ & $0.1(0.0,0.3)$ & $0.1(0.0,0.3)$ & $0.1(0.0,0.3)$ & $0.1(0.0,0.2)$ & $0.1(0.0,0.2)$ \\
\hline$\%$ time glucose $>180$ & $37(28,49)$ & $28(23,35)$ & $29(23,33)$ & $28(21,36)$ & $29(20,35)$ & $27(21,36)$ & $25(19,33)$ \\
\hline$\%$ time glucose $>250$ & $9(5,15)$ & $6(4,9)$ & $6(4,9)$ & $6(4,10)$ & $6(3,10)$ & $6(3,9)$ & $6(3,8)$ \\
\hline Mean glucose & $165(157,180)$ & $157(150,165)$ & $156(150,164)$ & $157(147,168)$ & $156(146,168)$ & $154(147,166)$ & $151(145,164)$ \\
\hline Glucose SD & $56(50,64)$ & $52(48,60)$ & $54(48,58)$ & $54(47,59)$ & $54(47,58)$ & $53(47,57)$ & $51(45,59)$ \\
\hline Glucose CV & $34(31,38)$ & $34(32,36)$ & $34(32,36)$ & $34(32,36)$ & $34(31,37)$ & $34(31,35)$ & $33(31,36)$ \\
\hline $\begin{array}{l}\text { \% time in closed-loop } \\
\text { when using CGM }\end{array}$ & $0.0(0.0,0.0)$ & $95(92,98)$ & $95(91,98)$ & $95(89,98)$ & $94(88,99)$ & $94(87,98)$ & $94(89,97)$ \\
\hline $\begin{array}{l}\% \text { time in closed-loop as } \\
\text { proportion of total time }\end{array}$ & $0.0(0.0,0.0)$ & $88(82,92)$ & $87(80,92)$ & $88(78,92)$ & $85(74,91)$ & $85(74,91)$ & $84(78,89)$ \\
\hline CGM use (\%) & $39(26,67) \S$ & $94(90,97)$ & $93(87,95)$ & $93(88,95)$ & $91(84,95)$ & $94(84,97)$ & $91(86,93)$ \\
\hline
\end{tabular}

Results presented as median (IQR). Glucose level are expressed in mg/dL. CGM, continuous glucose monitoring.

* Median duration of this period: 17.5 days (IQR: 10.5, 28).

$\dagger$ Trial duration was 26 weeks post randomization, irrespective of time to closed-loop activation. Some participants had longer duration due to insufficient masked CGM readings ¥ One participant was excluded from analysis due to withdrawal prior to closed loop activation

$\S$ CGM use between date of randomization and HCL activation. 